INVESTIGACIÓN

Recibido: 21/03/2021 --- Aceptado: 05/07/2021 --- Publicado: 28/07/2021

\title{
MIGRACIÓN DIGITAL EN LOS PROCESOS DE COMUNICACIÓN DE LAS TECNOLOGÍAS DE LA INFORMACIÓN Y LA COMUNICACIÓN (TIC) Y LOS MEDIOS DE COMUNICACIÓN SOCIAL
}

Digital migration in the communication processes of information and communication technologies (ICT) and the media of social communication

(D) $8 R^{6}$ Pastora Moreno-Espinosa: Universidad de Sevilla. España. pamoreno@us.es

(iD) $8 \mathrm{R}^{\mathrm{c}}$ " Aránzazu Román-San-Miguel: Universidad de Sevilla. España. arantxa@us.es

$8 R^{\circ}$ José-Israel Méndez-Ojeda: Universidad Autónoma de Yucatán. México. mojeda@correo.uady.mx

88 $\mathbb{R}^{6}$ Pedro Román Cu Acosta: Universidad Antropológica de Guadalajara. México pedro_aztec@yahoo.com.mx

Cómo citar el artículo:

Moreno-Espinosa, P., Román-San-Miguel, A., Méndez-Ojeda, J. I., y Cu Acosta, P. R. (2021). Migración digital en los procesos de comunicación de las tecnologías de la información y la comunicación (tic) y los medios de comunicación social. Vivat Academia. Revista de Comunicación, 69-88. http:// doi.org/10.15178/va.2021.154.e1361

http://www.vivatacademia.net/index.php/vivat/article/view/1361

\section{RESUMEN}

Este estudio pretende conocer las experiencias y el impacto producido por un blog desarrollado en la materia de Apreciación Musical. Los participantes fueron 20 estudiantes (entre adolescentes nativos digitales, y adultos) del nivel técnico, así como el profesor, migrante digital, cuya experiencia docente supera los 30 años en una institución de educación superior en Música Occidental en Jalisco, México. Como método se utilizó la Investigación Acción en el Aula, observaciones in situ, grupos focales, entrevistas en profundidad, diarios de campo y grabaciones de audio y video. Se concluye que la interacción mediada a través del blog en la enseñanza de la 
Moreno-Espinosa, P., Román-San-Miguel, A., Méndez-Ojeda, J. I., y Cu Acosta, P. R. Migración digital en los procesos de comunicación de las tecnologías de la información y la comunicación (tic) y los medios de comunicación social

Apreciación Musical es un componente motivacional cuyo diseño e implementación contribuyó a una mejor comprensión de los diferentes estilos musicales y las interacciones educomunicativas superaron las acciones de conocimiento tradicionales. Entre los resultados más relevantes de la investigación se encuentran los relacionados con la introducción de nuevos métodos y tecnologías en diferentes modalidades y entornos de aprendizaje, así como el estudio de las sinergias de los procesos de enseñanza-aprendizaje con los de comunicación, mediados por las diversas Tecnologías de la Información y la Comunicación (TIC) y los medios de comunicación social en las diferentes instituciones de educación superior y centros educativos dedicados a la enseñanza de la música, pertenecientes a la educación formal y no formal.

PALABRAS CLAVE: Investigación-acción - Blog - Migración digital - Interacciones del conocimiento - Aprendizaje-enseñanza - Estrategia - Apreciación musical Educomunicación - Competencias digitales.

\section{ABSTRACT}

This study aims to understand the experiences and the produced impact by a blog developed in Music Appreciation matter. Participants were 20 students (among digital native adolescents, and adults) of the technical level as well as the professor, digital immigrant, whose teaching experience exceeds 30 years in an institution of higher education in Western Music in Jalisco, Mexico. As method, it was used the Action Research in the Classroom, in situ observations, focus groups, depth interviews, field diaries, and audio and video recordings. It's concluded that the interaction mediated through the blog in the teaching of Music Appreciation is a motivational component whose design and implementation contributed to a better understanding of different musical styles and educommunicative interactions exceeded the traditional knowledge actions. Among the most relevant results of research are those related to the introduction of new methods and technologies in different modalities and learning environments, as well as the study of the synergies of the teaching-learning processes with the communication ones, mediated by the diverse Information and Communication Technologies (ICT) and social communication media in the different institutions of higher education and educational centers dedicated to the teaching of music, belonging to both formal and non-formal education.

KEYWORDS: Action Research - Blog - Digital Migration - Interactions of knowledge - Learning-Teaching - Strategy - Music Appreciation - Educommunication - Digital Skills.

Traducido por Paula González (Universidad Católica Andrés Bello, Venezuela) 
Moreno-Espinosa, P., Román-San-Miguel, A., Méndez-Ojeda, J. I., y Cu Acosta, P. R. Migración digital en los procesos de comunicación de las tecnologías de la información y la comunicación (tic) y los medios de comunicación social

\section{MIGRAÇÃO DIGITAL NOS PROCESSOS DE COMUNICAÇÃO DAS TECNOLOGIAS DA INFORMAÇÃO E COMUNICAÇÃO(TIC) E OS MEIOS DE COMUNICAÇÃO SOCIAL.}

\section{RESUMO}

Este estudo pretende conhecer as experiências e o impacto produzido por um blog desenvolvido na disciplina de apreciação musical. Os participantes foram 20 alunos (entre adolescentes nativos digitais, e adultos) do nível técnico, assim como o professor, imigrante digital cuja experiência supera os 30 anos em uma instituição de educação superior em música ocidental em Jalisco, México. Como método se utilizou a pesquisa de ação na sala de aula, observações in situ, grupos focais, questionários em profundidade, diários de bordo, e gravações de áudio e vídeo. Se conclui que a interação mediada através do blog no ensino de apreciação musical é um componente motivacional cujo design e implementação contribui a uma melhor compreensão dos diferentes estilos musicais e as interações educomunicativas superaram as ações de conhecimento tradicionais. Dentro dos resultados mais relevantes da pesquisa se encontram os relacionados com a introdução de novos métodos e tecnologias em diferentes modalidades e entornos de aprendizado, assim como o estudo das sinergias dos processos de ensino-aprendizado com os de comunicação, mediados pelas diversas tecnologias da informação e comunicação (TIC) e os meios de comunicação social nas diferentes instituições de educação superior e centros educativos dedicados ao ensino da música, que pertencem à educação formal e não formal.

\section{PALAVRAS CHAVE}

Pesquisa-ação - Blog - Migração digital - Interações do conhecimento - EnsinoAprendizado - Estratégia - Apreciação musical - Educomunicação - Competências digitais.

\section{INTRODUCCIÓN}

\subsection{Docentes migrantes digitales}

En la actualidad, existen diferentes perspectivas que explican que las Tecnologías de la Información y la Comunicación (TIC) originan un nuevo desafío en nuestros sistemas educativos, tanto por la transformación del modelo "tradicional" como por las modificaciones en las formas por las que los estudiantes adquieren o aprenden nueva información. Así, en el mundo actual, es necesario avanzar hacia un paradigma de educación diferente que incorpore contenidos, formas de enseñanza y aprendizaje tanto de docentes como de estudiantes en un proceso comunicativo distintivo utilizando las TIC, de tal manera que recuperen la educación como un acto natural de comunicación e incorporen como tema prioritario a estas tecnologías que forman parte del currículo escolar con espacios de reflexión sobre sus potencialidades, limitaciones y efectos en el aprendizaje (Ruthmann et al., 2015). También es evidente cómo los 
Moreno-Espinosa, P., Román-San-Miguel, A., Méndez-Ojeda, J. I., y Cu Acosta, P. R. Migración digital en los procesos de comunicación de las tecnologías de la información y la comunicación (tic) y los medios de comunicación social

sistemas escolares actuales enfrentan los requerimientos del mundo laboral que van más allá de las meras habilidades técnicas que demandan las TIC, ya que, según la Organización para la Cooperación y el Desarrollo Económicos (OCDE), en el siglo XXI, las habilidades más requeridas son habilidades cognitivas de orden superior, a través de las cuales se espera "la gestión del conocimiento, el análisis, la interacción y el intercambio de pensamiento creativo, crítico, reflexivo y colaborativo", que en su momento se han traducido en intentos fallidos de programas oficiales federales implementados en México como "Enciclomedia" y "Habilidades digitales para todos", constituidas únicamente en "conceptualizaciones tradicionales de la didáctica y versiones puramente transmisivas del aprendizaje" (Edel et al., 2015; OCDE, 2010). Por su parte, la Organización de las Naciones Unidas para la Educación, la Ciencia y la Cultura (UNESCO), a través de la División de Educación Superior, ha propuesto como uno de los pilares importantes de la globalización de la educación superior las innovaciones relacionadas con las TIC que tienen importantes consecuencias en este sector en términos de calidad, acceso, diversidad y financiamiento. También ha formulado pautas para el uso de las TIC por parte del profesorado, orientadas a transformar un entorno de aprendizaje centrado en el profesor en uno centrado en el alumno. Para ello, el organismo considera que los docentes deben tener acceso a una formación adecuada y oportunidades de desarrollo profesional constante para que estén motivados para aprender y aplicar nuevas técnicas y nuevos métodos de enseñanza y aprendizaje mediados por tecnologías (Semenov, 2005).

Una de las formas actuales de abordar esta necesidad en los docentes es analizar el papel del docente como migrante digital, como alguien con posibilidad de apropiarse de las tecnologías digitales y contribuir a la migración digital de otras personas, principalmente sus colegas, para ser un educador competente en sus áreas de contenido disciplinar, las tecnologías didácticas que utilizan para enseñar y seguir aprendiendo a medida que avanzan los cambios tecnológicos; reflexionar con ellos los procesos de migración digital que están a disposición del docente a través de un conjunto de condiciones, lineamientos, acciones y recursos que deben ser explotados de manera planificada, explícita y sistemática (González, 2013).

Así, hoy, los docentes migrantes digitales se enfrentan a la primera generación de estudiantes universitarios formados en nuevos avances tecnológicos rodeados de computadoras, videojuegos, entre otros, con habilidades de procesamiento de información y comunicación diferentes a las de la generación encargada de capacitarlos, (en este caso, docentes), estableciendo así una ruptura o brecha generacional que no se puede ignorar ni descuidar (Ayala \& Cassany, 2008; Prensky, 2010). Con la expansión de las nuevas tecnologías, los migrantes digitales tienen la oportunidad de evaluar las habilidades de los nativos digitales explorando diferentes formas y métodos de enseñanza y aprendizaje. El estudiante de hoy difícilmente encuentra atractivo una conferencia cara a cara a largo plazo o lecciones con lógica "paso a paso" y, a menudo, esto se interpreta como si el estudiante parece mostrar una falta de esfuerzo y seriedad durante su proceso de aprendizaje. Los llamados nativos digitales, a diferencia de los migrantes digitales, disfrutan de recibir información ágil e inmediata, prefieren los gráficos a los textos, se inclinan por los procesos aleatorios 
Moreno-Espinosa, P., Román-San-Miguel, A., Méndez-Ojeda, J. I., y Cu Acosta, P. R. Migración digital en los procesos de comunicación de las tecnologías de la información y la comunicación (tic) y los medios de comunicación social

(hipertexto), trabajan mejor y se desempeñan mejor cuando trabajan en la Web, y prefieren ser instruidos de forma lúdica en lugar de embarcarse en el rigor del trabajo tradicional (Prensky, 2010). El desafío para los docentes migrantes digitales es revalorizar su alfabetización digital para explorar seriamente el potencial de las diferentes herramientas tecnológicas, incluidas las redes sociales, e incorporar gradualmente las acciones enfocadas a la formación y práctica que forman parte de la era de la información y la comunicación, de manera que recuperen formas insospechadas de interacción relacionadas con su rol docente y se empoderen con las TIC, resultando en la mejora de los procesos de enseñanza-aprendizaje y la interacción social a través de las nuevas modalidades de enseñanza en la Web, potenciando así estos escenarios educativos según su especialidad, asignatura y nivel educativo (Davis et al., 2014).

\subsection{El empoderamiento de la educación musical con las TIC}

Hoy en día, la música de tradición escrita es accesible para un gran número de personas, sin diferencias étnicas, lingüísticas, de edad o de clase social. A diferencia de la antigüedad, la música y los artistas occidentales ahora se pueden difundir a través de los medios de comunicación, como la radio, internet y la televisión, lo que influyó en el aumento de las personas, primero, en el disfrute de la música y segundo, en el deseo de aprender a tocar un instrumento musical (Navarro et al., 2009). Es así como en nuestro tiempo, la influencia que ejercen los diferentes medios de comunicación e información social en la educación y difusión de la cultura, incluida la música, se debe en gran parte a las nuevas TIC, sin embargo, es real que la dedicación a su investigación en las diferentes artes está muy descuidada y desequilibrada en su tratamiento (Palomares, 2004).

Hoy en día, a los estudiantes les cuesta pensar en cómo era la vida cuando no existían los medios antes mencionados, incluso cambió el proceso de educación musical, ya que es necesario que esté a la par con lo que sucedió en las TIC (Sustaeta \& Domínguez, 2004). En general, la tecnología musical puede mejorar los procesos y reducir los tiempos de aprendizaje. Las herramientas informáticas, como los libros, los videos o la televisión, son un potencial agente mediador del aprendizaje cuyo uso depende de la visión musical del docente, así como de los principios que guían su acción educativa. Por ejemplo: el uso del vídeo como modalidad complementaria de presentación de información puede influir en el estudio de la digitación de determinados instrumentos musicales o técnicas de dirección orquestal (Tejada, 2004).

A partir de esta concepción de la música y su enseñanza sustentada en las TIC, el docente migrante generará su propio concepto de educación musical y diseñará un plan de acción que ponga en juego interacciones entre estudiantes, docente y mediadores (Tejada, 2004). Por ejemplo, en el caso de un grupo de la asignatura Dirección Orquestal de una institución de Educación Superior en el Reino Unido, el uso del video educativo por parte de los estudiantes significó un apoyo innovador y útil a la retroalimentación brindada por el docente, tanto de los ensayos como de los conciertos, porque les facilitó seguir las sugerencias del docente, y representó un 
Moreno-Espinosa, P., Román-San-Miguel, A., Méndez-Ojeda, J. I., y Cu Acosta, P. R. Migración digital en los procesos de comunicación de las tecnologías de la información y la comunicación (tic) y los medios de comunicación social

medio para retroalimentarse y observar mejoras en su técnica de dirección (Ramírez \& Sime, 2010). Entre otros escenarios mediados por las tecnologías destacan las aplicaciones didácticas para el ensayo coral virtual en el trabajo polifónico de grupos reducidos, en el que sus componentes pueden trabajar individualmente con el repertorio acordado con facilidades para ser descargados de internet en formato MIDI o mp3, y cada miembro del coro puede ensayar de forma independiente, ya sea con su voz individual o combinada con cualquiera de las otras voces, o con tonalidad (Sustaeta \& Domínguez, 2004).

Otro trabajo realizado, que involucra la enseñanza de las artes a través de las TIC, se refiere al estudio del canto. Se realizó una investigación cualitativa que consistió en la implementación de un curso experimental denominado "Aplicación de las nuevas tecnologías en la enseñanza del canto dirigido a 30 estudiantes", con diferentes grados de experiencia, desarrollo vocal y estilos de interpretación, cuyo objetivo fue verificar si la aplicación de las TIC en la enseñanza del canto facilita que los estudiantes obtengan resultados favorables durante su formación. Como parte de los resultados, se encontró que la inserción de programas informáticos permitió al docente, quien fungió como investigador participativo, calificar de manera objetiva el desarrollo de los estudiantes avanzados, quienes a su vez verificaron su avance a través del análisis de sus ecografías, comparadas con las de cantantes famosos a quienes tomaron como modelos. Los principiantes corrigieron deficiencias en el timbre, el ataque vocal y el apoyo respiratorio (Suárez, 2008).

Es notorio cómo la web, con sus diferentes herramientas, ha dibujado nuevos escenarios para la enseñanza y el aprendizaje de la música, proporcionando recursos extraordinarios para los educadores musicales de todos los ámbitos y niveles, y favoreciendo así el aprendizaje musical creativo, constructivo y significativo. En este sentido, la incorporación de las TIC en la enseñanza de la música ofrece la posibilidad de afrontar situaciones de aprendizaje variadas y enriquecedoras desde diferentes enfoques (Giráldez, 2006). En palabras de Wai-Chung (2009), el Internet se utiliza para explorar nuevos métodos de hacer música, composición e interpretación, junto con el análisis y discusión de sus aspectos culturales y la cultura digital.

Así, se puede afirmar que la cobertura de las TIC y los medios audiovisuales ha transformado el mundo de la música desde su producción, difusión y percepción. La web y sus recursos se han convertido en una fuente inagotable para investigar contextos en los que se desarrolla la música, promoviendo así el autoaprendizaje y el trabajo colaborativo donde estudiantes y docentes tienen la oportunidad de explorar nuevos escenarios que favorezcan el aprendizaje de la música, y reconocer la importancia de este empoderamiento tecnológico y sus aplicaciones en este campo (Sánchez, 2009).

\subsection{El blog y la enseñanza de la música}

A finales de los 90, nacieron los blogs para contrarrestar las limitaciones que se interponían en la comunicación entre el usuario del medio y el diseñador del contenido 
Moreno-Espinosa, P., Román-San-Miguel, A., Méndez-Ojeda, J. I., y Cu Acosta, P. R. Migración digital en los procesos de comunicación de las tecnologías de la información y la comunicación (tic) y los medios de comunicación social

online. Fue en 1997, cuando el desarrollador de software, emprendedor y escritor estadounidense Dave Winer crea el software necesario, y Jorn Barger crea el término Weblog al abrir una página personal en la web, considerado el primer blog verdadero y propio (Rojas, 2006).

El blog o weblog, es uno de los componentes de la "Web 2.0" y se ha convertido en los últimos años en un fenómeno dentro de ella. Se define como un diario personal online, "blog", un sitio web para recopilar y publicar mensajes o textos por uno o varios autores sobre un tema y cuyas historias se organizan en dos ejes: cronológico y temático. Es un espacio donde podemos crear un diario personal, donde el autor mantiene la libertad de publicar lo que considere oportuno (Seoane, 2007). Es una de las áreas más dinámicas de Internet, que se proyecta como un medio de comunicación online que busca un lugar entre las versiones electrónicas de los medios convencionales y digitales como portales de noticias, revistas, newsletters, entre otros tipos de información.

Las principales modalidades o denominaciones que adopta el blog son: el "edublog", como herramienta educativa para la docencia y la investigación; el "schoolblog", que es desarrollado en el entorno escolar por profesores y alumnos; el "audioblog", el "videoblog" y el "photoblog", que contienen archivos de audio, video y fotografías, respectivamente; y los "blogs de medios", que tratan sobre medios y comunicación. El blog se desarrolla dentro de una comunidad de usuarios que comparten un interés común; en general, el "blogger" es el responsable de su funcionamiento y realiza una serie de reflexiones $\mathrm{u}$ opiniones a través de los textos publicados, de manera que desde la educomunicación asume las funciones de docencia, comunicación, información, investigación, difusión, debate, organización, publicación de resultados científicos y educativos, entre otros (López \& Ballesteros, 2008).

El blog se ha utilizado para apoyar la práctica docente presencial, el desarrollo de unidades didácticas, aportar documentación adicional, enlazar a portales temáticos y otros espacios web, realizar trabajos de investigación y síntesis, exponer comentarios y dudas; un mayor empoderamiento del autoaprendizaje y la mayor participación de los estudiantes en la creación de materiales didácticos (Lima, 2008).

En cuanto a la investigación y experiencias con el blog en educación musical, está el trabajo desarrollado con estudiantes de pregrado de la asignatura de Análisis Musical en el Instituto Nacional de Educación, una institución de la Universidad Tecnológica de Nanyang en Singapur. Las habilidades musicales distribuidas se aprovecharon mediante el diseño de blogs. Se encontró que su implementación tiene un gran impacto pedagógico y motivacional como herramienta y estrategia en la enseñanza y el aprendizaje, fortalece el trabajo colaborativo y la organización de ideas durante este proceso (Chong, 2008).

En México, el Instituto de Investigación y Desarrollo Educativo de la Universidad Autónoma de Baja California (UABC), junto con la Escuela Nacional de Música de la 
Moreno-Espinosa, P., Román-San-Miguel, A., Méndez-Ojeda, J. I., y Cu Acosta, P. R. Migración digital en los procesos de comunicación de las tecnologías de la información y la comunicación (tic) y los medios de comunicación social

Universidad Nacional Autónoma de México (UNAM), realizó un estudio de investigación-desarrollo, que consistió en probar el blog como herramienta tecnopedagógica de enseñanza-aprendizaje para iniciar la interpretación de la Guitarra Clásica en un curso mixto, semipresencial o híbrido. Según los resultados, promovió actitudes favorables en los estudiantes, vinculadas a otras herramientas web como "YouTube" y generó un espacio para la gestación de los músicos, originando una forma de enseñar a tocar instrumentos musicales en línea, basada en modelos pedagógicos constructivistas (Navarro et al., 2009).

\section{OBJETIVOS}

Promover a través del blog prácticas innovadoras en las estrategias de enseñanza del docente como migrante digital y las estrategias de aprendizaje de los estudiantes, tanto nativos como migrantes digitales, en la asignatura Apreciación Musical dentro y fuera del aula.

Objetivos específicos del estudio:

1) Conocer las expectativas del docente y estudiantes de Apreciación Musical en relación a la inserción del blog para la enseñanza y el aprendizaje de la asignatura.

2) Diseñar e implementar un blog como auxiliar de las clases presenciales de Apreciación Musical.

3) Analizar con los alumnos y el docente el impacto del blog implementado en la asignatura de Apreciación Musical.

4) Reflexionar sobre cómo se produjo la transformación del proceso de enseñanza y aprendizaje (docente y alumnos) a través de la intervención innovadora mediada con el blog.

\section{METODOLOGÍA}

La investigación se realizó considerando el modelo de una institución pública del estado de Jalisco. En esta se enseña música occidental y su modelo equivale a las antiguas escuelas de música o conservatorios fundados en México hace más de 50 años, en cuyo sistema de enseñanza ha prevalecido la experiencia del maestro de música más que reconocidos métodos de enseñanza. La institución cuenta con una biblioteca que contiene libros, partituras y una biblioteca de sonido. Tiene conexión a internet limitada para profesores y alumnos oficiales. Los participantes de la investigación fueron 20 estudiantes del segundo semestre de 2012 en el plan de estudios de Técnico Musical en la asignatura de Apreciación Musical, estudiantes nativos y migrantes digitales, estudiantes de diversos instrumentos, con edades entre 15 y 35 años (100\% de los estudiantes). La escolaridad promedio transita entre secundaria y bachillerato, y una cantidad mínima tiene grado de educación superior. Trabajamos con el profesor de Apreciación Musical como migrante digital. 
Moreno-Espinosa, P., Román-San-Miguel, A., Méndez-Ojeda, J. I., y Cu Acosta, P. R. Migración digital en los procesos de comunicación de las tecnologías de la información y la comunicación (tic) y los medios de comunicación social

Esta investigación se llevó a cabo bajo el enfoque cualitativo, y el paradigma naturalista o interpretativo, donde una comunidad específica interpretó su realidad y necesidades particulares en un escenario, para luego elaborar una teoría derivada de ello (Pérez, 1990; Elliott, 2000). Se aplicó el proceso de investigación-acción participativa, donde la comunidad de nativos digitales y migrantes, junto con el investigador en su rol de participante, realizaron todas las interacciones. Este tipo de estudio se configura según un proceso cíclico que parte de la detección de una necesidad, y que una vez cubierta, concluye con su evaluación, lo que da lugar a un conocimiento emergente (Pérez, 1990) (Ver Figura 1). La intervención duró seis meses y el estudio se extendió a un año.

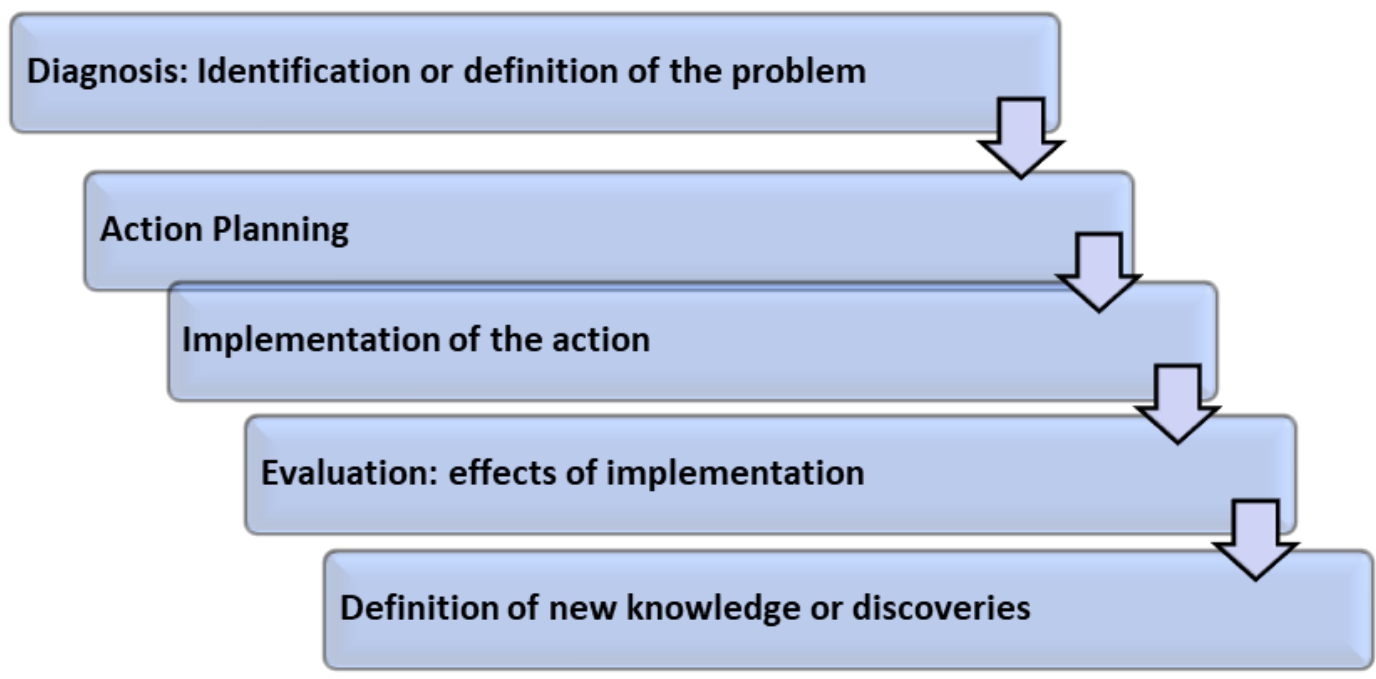

Figura 1. El ciclo Investigación-Acción

Fuente: basado en Pérez, 1990

Las técnicas de investigación para la recolección de datos que se utilizaron fueron: revistas de campo a través de las cuales se registraron las observaciones y apreciaciones del investigador; el análisis de documentos, ya que se revisó el temario de la asignatura junto con el libro de texto más utilizado por el docente; la entrevista en profundidad con el profesor migrante que nos brinda información en profundidad sobre sus experiencias como docente de apreciación musical y los grupos focales con los estudiantes que nos permiten indagar sobre los temas en la percepción de su actuación colectiva en audio y grabaciones de video. La confiabilidad diacrónica y la triangulación se utilizaron para garantizar la confiabilidad y validez de la investigación (Elliott, 2000; Galindo, 1999; Pérez, 1990). En cuanto al rol y la ética del investigador, actuó como moderador y asesor en las situaciones que lo requerían, fomentó el diálogo y mantuvo el anonimato de los participantes en el estudio.

\section{RESULTADOS Y DISCUSIÓN}

Desde la fase de diagnóstico, se indica que el docente asumió no conocer el blog y la forma de trabajarlo. Sin embargo, visualizó que su inserción en el aula podría generar interacciones ricas en experiencias en un escenario innovador que contribuya 
Moreno-Espinosa, P., Román-San-Miguel, A., Méndez-Ojeda, J. I., y Cu Acosta, P. R. Migración digital en los procesos de comunicación de las tecnologías de la información y la comunicación (tic) y los medios de comunicación social

a una mejor comprensión de la apreciación musical. Por su parte, los estudiantes (90\%) solicitaron la creación de un entorno virtual que les permita aprender desde cualquier punto fuera del aula y realizar tareas de escucha de melodías con mayor atención en horarios y espacios que se ajusten al tiempo y lugar disponible. Esto coincide con Aguaded et al. (2008), ya que el docente, al involucrarse con los estudiantes en los discursos de recursos tecnológicos, manifiesta un componente cognitivo y actitudinal favorable a ellos y proyecta su curso como parte del potencial de las TIC, integrando entonces las estrategias que se proponen desde, en, y por las comunidades educativas.

En este sentido, se destaca la oportunidad de los nuevos entornos formativos y sus beneficios para la formación y la comunicación, que posibilitan una propuesta viable en respuesta a necesidades con entornos flexibles, dinámicos, activos, interactivos, individualizados y colaborativos (Cabero \& Llorente, 2008), ya que el docente afirmó que "dos horas de clase presenciales semanales son insuficientes para analizar la música de forma que enriquezca el oído del alumno y, a su vez, este pueda distinguir la buena música de la mala". Además de eso, los estudiantes frecuentemente le solicitaban la grabación de CDs con melodías y también se expresaron respecto al uso del casete y reproductor de CDs en clase, lo cual les resultó monótono al tener que escuchar una pieza musical completa más de una vez en la que solo querían analizar fragmentos concretos de la misma, de modo que las funciones "adelante y atrás" en ese reproductor se realizaban con cierta imprecisión. Entonces, el blog es consistente con estas evidencias como oportunidad de superar los obstáculos que forman parte del acceso al contenido, contrarrestando así la pobreza que mantienen algunas instituciones dedicadas a la enseñanza de las artes en México en aspectos de infraestructura y equipamiento tecnológico (hardware y software) $(\mathrm{Cu}, 2012)$.

En cuanto a los componentes del blog, el profesor migrante y los estudiantes coincidieron en que los foros de discusión se deben utilizar para conocer las opiniones sobre la identificación de tonalidades, carácter, textura, períodos y estilos musicales, así como para publicar enlaces a "YouTube" sobre videos de melodías de reconocidos compositores de diferentes épocas. Además, los estudiantes (50\%) mencionaron la inserción de un chat donde todos pueden reunirse en un momento específico e interactuar para obtener retroalimentación y aclarar dudas. Por otro lado, se propuso que el blog funcione como un cronograma o planificación de actividades con sus temas y tareas, para acceder en caso de ausencia.

Como parte del plan de acción y la fase de implementación, se revisó el temario de la asignatura, se diseñó el blog y sus tareas de aprendizaje, se capacitó al docente y a los estudiantes migrantes digitales (ver figura 2) para acceder al blog y explotarlo para el propósito de la asignatura. La dirección web del blog es http:/ / apreciacionmusical.blogspot.mx/ (Zapata et al., 2014). 
Moreno-Espinosa, P., Román-San-Miguel, A., Méndez-Ojeda, J. I., y Cu Acosta, P. R. Migración digital en los procesos de comunicación de las tecnologías de la información y la comunicación (tic) y los medios de comunicación social

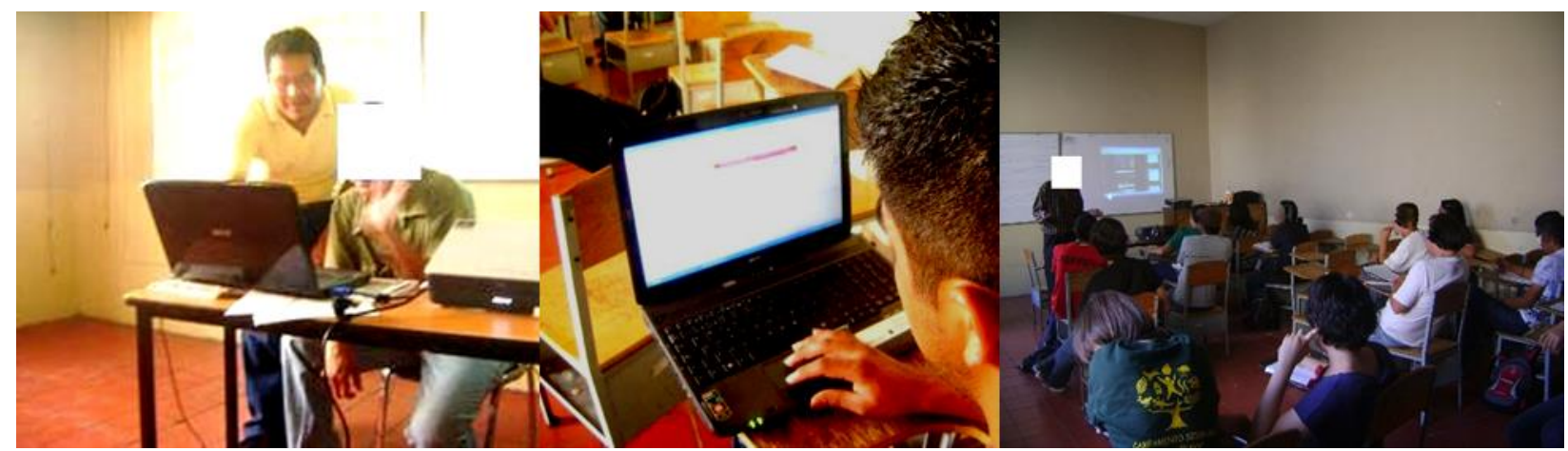

Figura 2. Formación de docentes y alumnos que participaron como migrantes digitales, y proyección del blog en el aula

Fuente: elaboración propia

En la evaluación de impacto del blog, los estudiantes (80\%) expresaron que presentaba un diseño adecuado y novedoso para la asignatura, que permitía un fácil acceso y uso. El profesor explicó que comprendió de inmediato los beneficios de implementar esta tecnología. También dijo que su diseño combinaba "aspectos muy clásicos como el barroco, con cosas modernas, haciendo que el blog tuviera un carácter a la vez serio y moderno". Asimismo, el uso de este medio tuvo para él un carácter motivador, ya que reconoce que el blog, en comparación con solo la música grabada, hubiera sido más laborioso lograrlo.

Tanto el profesor como los alumnos encontraron atractivo que el diseño les permitiera contribuir con videos y enlaces. Nuevamente, los estudiantes afirmaron que fue interesante tanto la proyección del blog en clase, como la revisión en casa de los ejercicios resueltos con el apoyo de los foros de discusión y videos de "YouTube" (80\%) porque esto provocó una variación de las interacciones en la clase y la dotó de "un nuevo tinte". A través de los foros, el docente reconoció y dio seguimiento a la participación consciente, y como valor agregado, le permitió conocer la opinión y personalidad de cada alumno, favoreciendo así un estrechamiento de la relación maestro-alumno. Por su parte, los estudiantes (90\%) también mostraron una gran aceptación hacia los foros de discusión, sin embargo, algunos de ellos observaron que en la medida en que los compañeros participaron, hubo respuestas idénticas, por lo que plantearon la posibilidad de que esas fueron el resultado de una mera copia y no de una auténtica evidencia de aprendizaje. Con esta última afirmación se puede decir que el alumno se ha involucrado en el ejercicio analítico de las potencialidades y limitaciones del blog $(\mathrm{Cu}, 2012)$.

En coincidencia con Cabero y Llorente (2008) quienes afirman que las TIC:

... nos ofrecen una serie de posibilidades que las hacen verdaderamente útiles para su incorporación a los entornos formativos: ampliación de la oferta informativa, creación de entornos más flexibles para el aprendizaje, eliminación de barreras espacio-temporales para la interacción entre profesor y alumnos, aumento en modalidades de comunicación, potenciación de escenarios y entornos interactivos, favoreciendo tanto el aprendizaje autónomo como el 
Moreno-Espinosa, P., Román-San-Miguel, A., Méndez-Ojeda, J. I., y Cu Acosta, P. R. Migración digital en los procesos de comunicación de las tecnologías de la información y la comunicación (tic) y los medios de comunicación social

aprendizaje colaborativo ... [...] ... nuevas formas de organizar las actividades docentes ... [...] ... creando entornos diferenciados adaptados a las características cognitivas de los estudiantes (p. 8-9).

En cuanto a los videos de "YouTube", el docente reconoció el gran valor educativo que tiene si cada profesor de música dedica tiempo a la búsqueda y selección de contenidos fiables. Para los estudiantes, fue una herramienta útil a la hora de realizar sus tareas de forma flexible en sustitución del casete y el reproductor de CD. Cada alumno podía reproducir la pieza musical completa más de una vez o centrar su atención en los fragmentos de melodías que les parecieran más interesantes de identificar o analizar en un ejercicio de aprendizaje individual $(\mathrm{Cu}, 2012)$.

El profesor agregó que logró captar la atención y el compromiso de sus alumnos, incluidos aquellos que no mostraron interés en escuchar música de concierto al inicio del semestre, utilizando el blog dentro del aula con la ayuda del equipo de audio y proyección prestado, para que pudiera usar las partes del blog en las que estaba interesado en enfatizar más. Además, reconoció el cambio en la forma de formular instrucciones en el blog gracias al diseño instruccional, lo que provocó brindar una mejor orientación a sus alumnos quienes dijeron que las instrucciones eran claras y bien estructuradas. En este sentido, Beloch (2013) indica que el diseño instruccional para trabajar en entornos virtuales es una tarea ardua, que requiere una formación en esta materia que permita el uso de las modalidades de enseñanza-aprendizaje a través de la web e implica contemplar tanto al docente como al alumno, la modalidad docente, los objetivos del programa, las tareas, ejercicios y materiales, entre otros.

Por otro lado, el blog en la enseñanza de la apreciación musical cumple con su finalidad didáctica educativa. Es una herramienta amigable e intuitiva y se puede utilizar tanto dentro como fuera del aula en un trabajo colaborativo, donde el alumno toma el control de su aprendizaje (Cabero \& Llorente, 2008) en un innovador enfoque pedagógico del trabajo para una modalidad de curso mixto. En relación a las debilidades detectadas en el uso de los foros de discusión, los estudiantes sugirieron que se podría mejorar su formalidad y las tareas, con una plataforma que permita diseñarlas con anticipación y programarlas con fechas próximas, de cierre o límites de publicación y realización para lograr un mayor control y la prevención de participaciones desorganizadas. En cuanto al chat que se propuso, se utilizó con poca demanda porque era difícil para los estudiantes acordar una hora para reunirse y discutir. Por otro lado, el docente destacó que el blog superó sus expectativas iniciales, pues reconoció que llegó a acercar a sus alumnos a la música $(\mathrm{Cu}, 2012)$.

A efectos de evaluación de la asignatura, el blog permitió una consulta más cómoda para corroborar el avance, participación y logros de los estudiantes, ya que el docente reconoció que hubiera sido difícil lograrlo apoyándose únicamente en la lista de asistencia impresa y asignando en él "notas cuantitativas o cualitativas sobre el desempeño de cada estudiante". A los estudiantes se les permitió realizar propuestas, entre ellas el hecho de que los estudiantes solicitaran mayor diversidad de materiales para este fin, que también complementan sus actividades presenciales, principalmente 
Moreno-Espinosa, P., Román-San-Miguel, A., Méndez-Ojeda, J. I., y Cu Acosta, P. R. Migración digital en los procesos de comunicación de las tecnologías de la información y la comunicación (tic) y los medios de comunicación social

para el inicio del aprendizaje de instrumentos musicales. Otros pedidos fueron la creación de una biblioteca sonora virtual, en la que se puede descargar un repertorio musical de diferentes géneros y estilos musicales para analizar desde sus casas, una biblioteca virtual que contenga libros sobre música, y principalmente, partituras para cada instrumento. Argumentaron que, en ocasiones, cuando acudían a la biblioteca, el material que solicitaban no estaba disponible, y les resultaría más fácil acceder a este contenido al descargarlo desde una plataforma tecnológica desde sus hogares. También demandaron materiales de audio en formato mp3 para el estudio de los diferentes acordes, dictados rítmico-melódicos, así como el diseño de tutoriales que refuercen el aprendizaje de ciertos instrumentos musicales en los aspectos de postura, técnica de ejecución, entre otras habilidades. Además, el docente sugirió que sería muy útil contar con una colección de videos de capacitación sobre clases magistrales de reconocidos instrumentistas y cantantes, para no depender únicamente de estas clases presenciales (Zapata et al., 2014).

\section{CONCLUSIONES}

Con base en los resultados y su análisis, se puede concluir que el proceso de migración digital conduce a interacciones complejas en el docente migrante, lo que lo lleva a reflexionar y mejorar su propia práctica en el escenario convencional y web, en una transformación tanto de la comunicación como de la procesos de enseñanza y aprendizaje, que incluye a estudiantes más motivados a aprender mediante la inserción de modalidades innovadoras que no pueden ser utilizadas con un determinismo puramente tecnológico. Esto se relaciona con la tendencia a enfocarse solo en la apariencia y el punto de vista técnico de los dispositivos, criterios en su mayoría promovidos por las industrias tecnológicas, el marketing, la publicidad y el mundo del entretenimiento, (Bauman, 2017) y cuyas influencias se traducen en aplicaciones incorrectas de la tecnología. Para superar esta situación, los educadores pueden desarrollar un sentido de evaluación crítica y reflexiva cuando estas tecnologías forman parte de los procesos pedagógicos que se llevan a cabo en el aula, ya que estamos inmersos en un mundo donde "es difícil trazar una línea divisoria clara entre la humanidad musical y la humanidad tecnológica" y el estudio de las prácticas y significados sociales ha sido abandonado como parte de la llamada "cultura participativa" en la que ha intervenido la tecnología (Himonides, 2012; Ruthmann et al., 2015).

La metodología de investigación-acción en su carácter participativo permite la cooperación y la reconstrucción del conocimiento de la comunidad migrante, nativos digitales, el investigador y la comunidad escolar, ya que esto permite interacciones enriquecedoras y espacios de reflexión crítica que incentivan la formulación de alternativas de trabajo con las TIC en una dinámica de innovación educativa para la enseñanza de la apreciación musical.

Es importante señalar que la metodología de la investigación aplicada fue producto de la participación conjunta de una comunidad escolar, tanto en la construcción colectiva del problema y las necesidades, como en los planes de acción realizados para 
Moreno-Espinosa, P., Román-San-Miguel, A., Méndez-Ojeda, J. I., y Cu Acosta, P. R. Migración digital en los procesos de comunicación de las tecnologías de la información y la comunicación (tic) y los medios de comunicación social

contribuir a su solución. La formulación del plan de acción como parte vital del estudio es una construcción del colectivo en función de sus necesidades formativas en la materia y requerimientos de acuerdo a sus conocimientos en nuevos escenarios, competencias informáticas y la versatilidad de herramientas en la web 2.0, en este caso el trabajo con el blog.

Es posible innovar en el aula apoyado en las TIC y esto dependerá de la visión que el docente tenga de la enseñanza de la música, así como del desarrollo de actitudes, tanto del docente como de los alumnos, para incentivar el uso de estas nuevas modalidades con fines pedagógicos y didácticos a pesar de las limitaciones, ya que las posibilidades ante la amplia variedad de plataformas y herramientas gratuitas de la Web 2.0 comprueban que tienen versatilidad y flexibilidad que permiten un control diferente e innovador en la participación de docentes y alumnos, donde ambos asumen su propio control y construcción de sus propios procesos de formación y conocimiento con el fin de optimizar sus procesos a la hora de enseñar y aprender en el aula. Ese fue el caso de la materia de apreciación musical. De hecho, el 90\% de los estudiantes considera que la participación en el blog les ayudó a expresarse con mayor facilidad que en el aula, reduciendo así el estrés, y en el caso de aquellos que tienden a expresar abiertamente sus opiniones en clase, podrán para conocer la opinión de otros compañeros a través del blog.

Se recomienda para propósitos de futuras investigaciones, que en los conservatorios y escuelas de educación musical en México se realicen estudios empíricos que sean sistemáticos, precisos y oportunos sobre las alfabetizaciones digitales de sus docentes y alumnos. También es recomendable investigar en nuestro país las prácticas con diferentes dispositivos, aplicaciones móviles, los diversos usos de los medios audiovisuales, la televisión como herramienta educativa y cultural, todo ello para profundizar en el grado de autenticidad de los aprendizajes, para comprender los procesos de comunicación (en qué medida las TIC y las redes sociales funcionan como mediadores del conocimiento en los escenarios de enseñanza y aprendizaje de música y son utilizados para tales fines por docentes, estudiantes y músicos en general). Otros problemas que pueden ser investigados en cuanto al uso de estas herramientas tecnológicas y medios de comunicación social a través de estudios empíricos consistentes de carácter interdisciplinario son: los procesos cognitivos que se generan, el desarrollo de diversas habilidades, patrones de comportamiento social, procesos socioafectivos e identidades, así como la descripción de imaginarios sociales entre los diferentes actores educativos y sociales involucrados, el capital social y cultural de los estudiantes y profesores de música, el papel mediador que desempeñan en las industrias culturales, entre otros posibles objetos de estudio. Esto permitirá, por un lado, un punto de partida sólido y firme para orientar la formación integral de los estudiantes de las comunidades escolares de arte, y por otro, detectar necesidades en cuanto a infraestructura e instalaciones para avanzar en soluciones viables. También se sugiere realizar investigaciones sobre temas relacionados con la evaluación curricular interna de los programas educativos para que la inserción de las TIC en esta área sea relevante y tenga un impacto positivo en los procesos de enseñanza y 
Moreno-Espinosa, P., Román-San-Miguel, A., Méndez-Ojeda, J. I., y Cu Acosta, P. R. Migración digital en los procesos de comunicación de las tecnologías de la información y la comunicación (tic) y los medios de comunicación social

aprendizaje, así como en la formación docente (Davis et al., 2014; Grimshaw, 2014; McPherson y Welch, 2012; Ruthmann et al., 2015; Reeves et al., 2017; Semenov, 2005).

Finalmente, es necesario evaluar, antes de implementar, el potencial de las diferentes herramientas que ofrece Internet con fines educativos, incluyendo los Sistemas de Gestión del Aprendizaje, los diferentes Sistemas de Gestión de Contenidos y las diferentes herramientas Web 2.0, para determinar cuáles son las más adecuadas en el estudio de un instrumento musical específico, así como de las materias teóricas, musicales, pedagógicas y/o metodológicas. Los contenidos derivados de las herramientas funcionales servirán para enriquecer y potenciar las funciones de los Sistemas de Gestión del Aprendizaje que gestionan las instituciones de la música. Por tanto, será necesario impulsar políticas internas para el mejor uso de las TIC en las mismas entidades.

\section{REFERENCIAS}

Aguaded Gómez, J. I., Tirado Morueta, R., \& Cabero Almenara, J. (2008). Los centros TIC en Andalucía, España: un modelo de implicación del profesorado en la integración curricular de la tecnología. SOCIOTAM, 17(2), 171-199.

Ayala, G., \& Cassany, D. (2008). Nativos e inmigrantes digitales en la escuela. Revista CEE Participación Educativa, 9, 53-71. https://cutt.ly/umht08k

Bauman, Z. (2013). Sobre la Educación en un mundo líquido. Conversaciones con Ricardo Mazzeo (D. Payás Puigamau, Trad). Paidós.

Beloch, C. (2013). Diseño Instruccional, Entornos virtuales y de formación. Recursos tecnológicos en Educación y Logopedia. Universidad de Valencia. http://www.uv.es/bellochc/pedagogia/EVA4.wiki?7

Cabero Almenara, J., \& Llorente Cejudo, M. C. (2008). La alfabetización digital de los alumnos. Competencias digitales para el siglo XXI. Revista Portuguesa de Pedagogía, 42(2), 7-28. http://tecnologiaedu.us.es/cuestionario/bibliovir/jca26.pdf

Chong, E. K. M. (2008). Harnessing distributed musical expertise through edublogging. Australasian Journal of Educational Technology, 24(2), 181-194. http://ajet.org.au/index.php/AJET/article/view/1220/446

Cu Acosta, P. R. (2012). Plataforma Tecnológica como estrategia de enseñanza y aprendizaje de Apreciación Musical del Mundo Occidental: una experiencia con el blog. [Tesis doctoral, Universidad Autónoma de Yucatán]. https://cutt.ly/JmhyyzD

Davis, C., Deil-Amen, R., Rios-Aguilar, C., \& González, M. (2014). Social Media, Higher Education, and Community Colleges: Research Synthesis and Implications 
Moreno-Espinosa, P., Román-San-Miguel, A., Méndez-Ojeda, J. I., y Cu Acosta, P. R. Migración digital en los procesos de comunicación de las tecnologías de la información y la comunicación (tic) y los medios de comunicación social

for the Study of Two-Year Institutions, Community College. Journal of Research and Practice. https://doi.org/10.1093/oxfordhb/9780199826162.001.000110.1080

Elliott, J. (2000). El cambio educativo desde la investigación acción. (5th edition). Morata.

Galindo Cáceres, L. J. (1999). Técnicas de investigación en sociedad, cultura y comunicación. Pearson Addison Wesley Longman.

Giráldez Hayes, A. (2006). Internet y Educación Musical. Grao.

González Gartland, G. (2013). ¿Incluidos, formados y capacitados? El impacto de las migraciones digitales en el rol docente. In Cabello, R. Migraciones digitales: comunicación, educación, y tecnologías digitales interactivas, (pp. 79-100) Universidad Nacional de General Sarmiento. https://cutt.ly/0mhi2C0

Grimshaw, M. (2014). The Oxford handbook of virtuality. University Press. https:// doi.org/10.1093/oxfordhb/9780199826162.001.0001

Himonides, E. (2012). Music Learning and teaching through technology. In McPherson, G. and Welch, G. (eds.) The Oxford Handbook of Music Education, (2). Oxford Press. https://doi.org/10.1093/oxfordhb/9780199928019.013.0028

Lima Torrado, J. (2008). Utilización de weblogs como instrumento de educación en Derechos Humanos dentro de la plataforma Webct de la Universidad Complutense de Madrid en el contexto del Proceso de Convergencia Europea de Educación Superior. IV Jornada Campus Virtual UCM: Experiencias en el Campus Virtual (pp. 111115). Editorial Complutense. http://eprints.ucm.es/7798/1/campusvirtual118122.pdf

López Meneses, E., \& Ballesteros Regaña, C. (2008). Caminando hacia el software social: Una experiencia universitaria con blogs. Pixel Bit. Revista de Medios y Educación, 32(5), 67-82. https://cutt.ly/CmhoQy8

McPherson, G., \& Welch, G. (2012). The Oxford Handbook of Music Education. Oxford University Press.

Morales-González, B., Edel-Navarro R., \& Aguirre-Aguilar, G. (2015). Metacognición y tecnologías de la información y la comunicación: coincidencias e inconsistencias en la investigación. Sinéctica: Revista Electrónica de Educación, 45. https:// sinectica.iteso.mx/index.php/SINECTICA/article/viewFile/592/585

Navarro, J. L., Lavigne, G., \& Martínez Salgado, G. G. (2009). Curso de Guitarra Clásica en línea: blogs para la enseñanza musical. Revista Electrónica LEEME (Lista Electrónica Europea de Música en la Educación), 24. https://ojs.uv.es/index.php/LEEME/article/view/9794 
Moreno-Espinosa, P., Román-San-Miguel, A., Méndez-Ojeda, J. I., y Cu Acosta, P. R. Migración digital en los procesos de comunicación de las tecnologías de la información y la comunicación (tic) y los medios de comunicación social

OCDE (2010). Are the new millennium learners making the grade? Technology use and educational performance in PISA 2006. OECD Multilingual Summaries. http://www.oecd.org/edu/ceri/45053490.pdf

Palomares Moral, J. (2004). Comunicar la música. Comunicar, 23, 13-16. http://www.redalyc.org/pdf/158/15802303.pdf

Pérez Serrano, G. (1990). Investigación Acción: Aplicaciones en el campo social y educativo. Dykinson.

Prensky, M. (2010). Nativos e inmigrantes digitales. Distribuidora SEK, S. A. https://cutt.ly/hmhoBV1

Ramírez Martinell, A., \& Sime, J. (2010). Video enriched learning experiences for performing arts students: two exploratory case studies. In O'Donoghue, J. (Ed.), Technology Supported Environment for Personalised Learning: Methods and Case Studies (pp. 248-267). IGI Global.

Reeves, T., Caglayan, E., \& Torr, R. (2017). Don't shoot! understanding students' experiences of video-based learning and assessment in the arts. Video Journal of Education and Pedagogy, 2(1). https://videoeducationjournal.springeropen.com/articles/10.1186/s40990-016$\underline{0011-2}$

Rojas Orduña, O. I. (2006). Blogs: La conversación en internet que está revolucionando medios, empresas, politicos y ciudadanos. ESIC.

Ruthmann, S. A., Tobías, E. S., Randles, C. \& Thibeault, M. (2015). Is it the technology? Challenging technological determinism in music education. In Randles C. (Ed.), Music Education: Navigating the future (pp.122-138). NY Routledge.

Sánchez Llamas, J. (2009). Los objetivos del currículo musical y su puesta en práctica en los I. E. S. Temas para Educación: Revista Digital para profesionales de la Enseñanza. Federación de Enseñanza de CC.OO. de Andalucía, 4. http:/ / www.feandalucia.ccoo.es /indcontei.aspx?d=3423\&s=5\&ind $=177$

Semenov, A. (2005). Las Tecnologías de la Información y la Comunicación en la enseñanza. Manual para docentes. UNESCO. http://unesdoc.unesco.org/images/0013/001390/139028s.pdf

Seoane García, C. (2007). Blogs, los nuevos colegios invisibles. (Espacios de creación, diálogo y aprendizaje). Cuadernos de Biblioteconomia Arquivística e Documentação Cadernos $B A D$, 23-37. http://www.apbad.pt/CadernosBAD/Caderno12007/CGarciaCBAD107.pdf 
Moreno-Espinosa, P., Román-San-Miguel, A., Méndez-Ojeda, J. I., y Cu Acosta, P. R. Migración digital en los procesos de comunicación de las tecnologías de la información y la comunicación (tic) y los medios de comunicación social

Suárez Mazón, E. M. (2008). Aplicación de Teorías Pedagógicas Modernas y Recursos Informáticos en la Enseñanza del Canto: Antecedentes Históricos. [Tesis doctoral, Universidad Carlos III de Madrid]. http:/ / dialnet.unirioja.es/servlet/tesis?codigo=18772

Sustaeta, I., \& Domínguez-Alcahud, M. P. (2004). Aplicaciones didácticas de la informática musical. Revista Electrónica Complutense de Investigación en Educación Musical, (4). http://pendientedemigracion.ucm.es/info/reciem/v1n4.pdf

Tejada Giménez, J. (2004). Música y mediación de la tecnología en sus procesos de aprendizaje. Educación XXI Revista de la Universidad Nacional de Educación a Distancia, 7. http://www.redalyc.org/pdf/706/70600701.pdf

Tobias, E. (2015). Inter/trans/cross/new media(ting): Navigating and emerging landscape of digital media for music education. In Randles, C. (Ed.) Music Education: Navigating the future (pp.91-121). NY Routledge.

Wai-Chung, H. (2009). The role of multimedia technology in a Hong Kong Higher Education Music program. Visions of Research in Music Education, 13(1). http://www-usr.rider.edu/ vrme/v13n1/Vision/Ho.finalfaedits.01.15.09.pdf

Zapata González, A., Méndez Ojeda, J. I., \& Cu Acosta, P. R. (2014). Una nueva forma de enseñar y aprender la apreciación musical utilizando el blog. In Prieto Méndez M. E.; Pech Campos S. J.; De León, T. and García, J. F. Technologies and Learning: Innovation and Experiences (pp. 625-630). Humboldt International University/CIATA.

\section{AUTOR/ES:}

\section{Pastora Moreno-Espinosa:}

Catedrática del Departamento de Periodismo II de la Universidad de Sevilla. Ha sido profesora visitante en diversas Universidades: Bruselas, Bolonia, Milán Verona, Universidad Nacional de Chengchi en Taipei (República China de Taiwan), Universidad de Mérida-Yucatán (México), Universidad de las Américas (Puebla), Universidad Autónoma de Chihuahua, Universidad de San Salvador, Radiotelevisión de Veracruz, Universidad Veracruzana, Universidad Anáhuac de Cancún, Universidad de La Habana, etc. Es Directora del Equipo de Investigación en Géneros Audiovisuales e Imágenes, EGAUDIM. Ha colaborado en diferentes medios de comunicación, tanto de Prensa como de Radio y Televisión y en numerosos Seminarios, Simposios y Congresos nacionales e internacionales.

Orcid ID: https:// orcid.org/0000-0001-6494-130X

Google Scholar: https://scholar.google.es/citations?user=GFGjFrsAAAAJ\&hl=es Research ID: https://publons.com/researcher/1939741/pastora-moreno-espinosa/ Scopus: https:// www.scopus.com/authid/detail.uri?authorId=51663715200 
Moreno-Espinosa, P., Román-San-Miguel, A., Méndez-Ojeda, J. I., y Cu Acosta, P. R. Migración digital en los procesos de comunicación de las tecnologías de la información y la comunicación (tic) y los medios de comunicación social

Researchgate: https://www.researchgate.net/scientific-contributions/Dra-PastoraMoreno-Espinosa-2033866337

Dialnet: https:/ / dialnet.unirioja.es/servlet/autor?codigo=761369

Idus: https://idus.us.es/author-profiles/author?id=2815

\section{Aránzazu Román-San-Miguel:}

Es Profesora Ayudante Doctora y ayudante de dirección del Máster en Periodismo Deportivo en la Universidad de Sevilla, además de miembro del Grupo de Investigación: Estrategias de Comunicación. Doctora en Periodismo, es Licenciada en Periodismo y Experta Universitaria en Comunicación Institucional y Marketing Político. Ha sido profesora y co-coordinadora del Máster en Comunicación Institucional y Política en la universidad hispalense. Ha escrito varios libros, capítulos de libro y artículos en revistas. Ha realizado estancias de investigación en la Universidad Complutense de Madrid, en la Universidad de Castilla La Mancha y en la Universidad Pontificia Salesiana de Roma.

Orcid ID: https:// orcid.org/0000-0002-9131-2629

Google Scholar: https://scholar.google.com/citations?user=KQIS_z0AAAAJ\&hl=es

Research ID: https://publons.com/researcher/2279421/aranzazu-arsm-roman-san$\underline{\text { miguel }}$

Scopus: https:// www.scopus.com/authid/detail.uri?authorId=56041639800

Researchgate: https://www.researchgate.net/profile/Aranzazu_Roman_San_Miguel Dialnet: https://dialnet.unirioja.es/ servlet/autor?codigo=2598513

Idus: $\quad$ https://idus.us.es/browse?value=Rom\%C3\%A1n-SanMiguel, \%20Ar\%C3\%A1nzazu\&type=author

\section{José-Israel Méndez-Ojeda}

Es Doctor en Comunicación y Cultura en la Sociedad de la Información por parte de la Universidad de Sevilla. Profesor e investigador en las líneas de Educomunicación, TICs, Sociología educativa, Microsociología y Actuaciones en la educación. Es miembro del Cuerpo Académico Consolidado de Currículum e Instrucción. Perfil Deseable del programa de mejoramiento de profesorado SEP. Imparte las asignaturas de Análisis microsociológico y comunicativo de las actuaciones en la escuela, Taller de habilidades docentes, Materiales de instrucción, Televisión educativa, Dirección y Producción del audiovisual educativo.

Orcid ID: https:/ / orcid.org/0000-0002-0335-0423

Google Scholar: https://scholar.google.es/citations?user=V0nFjvcAAAAJ\&hl=es

Researchgate: https://www.researchgate.net/profile/Jose-Ojeda-4

Idus:

https://idus.us.es/browse?value=M\%C3\%A9ndez\%20Ojeda, \%20Jos\%C3\%A9\%20Isr ael\&type=author

\section{Pedro Román Cu Acosta}

Es Maestro en Innovación Educativa y Licenciado en Educación por la Universidad Autónoma de Yucatán. Actualmente es profesor en la Universidad Antropológica de Guadalajara (México) y en la Universidad Virtual de Estudios Superiores. Su línea de 
Moreno-Espinosa, P., Román-San-Miguel, A., Méndez-Ojeda, J. I., y Cu Acosta, P. R. Migración digital en los procesos de comunicación de las tecnologías de la información y la comunicación (tic) y los medios de comunicación social

investigación se centra en la innovación pedagógica y tecnológica, así como la alfabetización mediática en el ámbito de la enseñanza de música.

Google Scholar: https://scholar.google.es/citations?user=C05d9FgAAAAJ\&hl=es

Researchgate: https://www.researchgate.net/profile/Pedro-Cu-Acosta 